\title{
Fabrication of three-dimensional microstructures by soft molding
}

\author{
Y. S. Kim, K. Y. Suh, and Hong H. Lee ${ }^{\text {a) }}$ \\ School of Chemical Engineering, Seoul National University, Seoul, 151-742 Korea
}

(Received 29 May 2001; accepted for publication 27 July 2001)

\begin{abstract}
We have developed soft molding as a method for meso-scale-area fabrication of three-dimensional structures. The soft molding, which is a form of soft lithography, involves placing an elastomeric mold on the surface of a spin-coated polymer film with a slight pressure $\left(<1 \mathrm{~N} / \mathrm{cm}^{2}\right)$, allowing the mold to absorb solvent, releasing the pressure, and then letting the mold and the substrate remain undisturbed for a period of time. The three-dimensional structure thus formed is robust in that the pattern fidelity is preserved without any distortion or defects. For the soft molding to be successful, the rate of solvent absorption by the mold should be larger than the rate of solvent evaporation. The method is demonstrated with several three-dimensional structures. (C) 2001 American Institute of Physics. [DOI: 10.1063/1.1407859]
\end{abstract}

Three-dimensional microscale patterning that nontraditional lithographies allow is a major advantage over more costly conventional photolithography methods. These nontraditional lithographies involve the use of a mold. When the mold is soft, the material consists of elastomers, and the lithography method is referred to as soft lithography. ${ }^{1-4}$ When the mold is hard, usually made out of silicon wafers, it is called imprint lithography. ${ }^{5}$ Imprint lithography involves applying a very high pressure $\left(3000-15000 \mathrm{~N} / \mathrm{cm}^{2}\right)$ for the imprinting of the mold pattern into an underlying polymer layer. Some apparent limitations ${ }^{6}$ inherent in the imprint lithography make it somewhat unsuitable for the formation of three-dimensional microstructures. In fact, no such result has been reported in the literature. On the other hand, the soft lithography has been used in the form of solvent-assisted microcontact molding (SAMIM) (Refs. 2 and 3) and micromolding ${ }^{4}$ for the purpose of fabricating threedimensional structures. Three-dimensional patterning can find immediate applications in micro-optics ${ }^{7}$ and microelectromechanical systems.

We report here a method for forming three-dimensional microstructures that overcomes the difficulties and shortcomings of SAMIM and micromolding. In SAMIM, a polymer film is coated onto a substrate and baked. An elastomeric mold with relief patterns, usually made from poly (dimethylsiloxane) (PDMS), is wetted with a good solvent for the polymer, which is then brought into contact with the polymer surface. The solvent on the mold dissolves the polymer and this dissolved polymer is molded into the channels or cavities formed between the mold and the substrate. There are two problems with SAMIM when it is applied to forming three-dimensional structures. The first is that the surface of the patterned polymer is highly textured, which may be useful in processes where a high surface/volume ratio is important, as in adhesion and catalysis, but is not desirable at all for forming three-dimensional structures. The other is that the pattern formed by SAMIM gets distorted. In particular, the base of a rectangular stripe becomes wider than the top.

\footnotetext{
a) Author to whom correspondence should be addressed; electronic mail: honghlee@plaza.snu.ac.kr
}

Furthermore, the structure formed contracts as the solvent evaporates from the molded polymer.

The same problem arises in the method of micromolding, ${ }^{4}$ which was used to fabricate glass microstructures from sol-gel precursors. The shrinkage problem is further amplified by isotropic shrinkage caused by the annealing step required for the fabrication. For the method to work, there has to be regions where the material to be molded is not present so that the elastomeric mold and the bare substrate are allowed to make contact. Generation of defects is another problem to contend with.

The problems associated with SAMIM and micromolding in forming three-dimensional structures are essentially solvent related. If a polymer film spin coated onto a substrate is directly molded by an elastomeric mold without baking, the solvent concentration in the film is initially uniform, relieving any problem associated with the initial nonuniform distribution of solvent. If a solvent is appropriately chosen such that the solvent is readily absorbed into the elastomeric mold and evaporates into air by diffusion through the mold, the fidelity of the structure being molded can be assured. Use of a solvent with efficient absorption and dissipation characteristics and that of a spin-coated polymer film without baking are the two essential features of the method called "soft molding," which is a form of soft lithography.

Soft molding was motivated by our previous work on imprint lithography by solvent vapor treatment. ${ }^{8}$ We wanted to substantially reduce the high pressure still needed by the solvent vapor treatment by making the polymer film laden with a sufficient amount of solvent. Use of a hard mold that is typical of the imprint lithography for such a film led to no pattern formation. The idea was then to use a soft mold that is capable of absorbing the solvent such that by the time the polymer is molded, the film does not contain much solvent.

Figure 1 illustrates the soft molding process. An elastomeric mold with the desired pattern on its surface is placed onto a polymer film, without baking, immediately after the film is spin coated onto a substrate and then pressed slightly at a pressure of less than $1 \mathrm{~N} / \mathrm{cm}^{2}$. After releasing the pressure, the mold and the substrate are allowed to remain undisturbed for a period of time, which is on the order of 10 


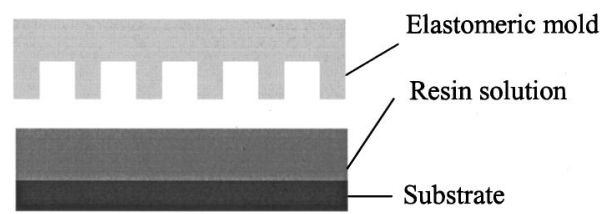

Slight pressing

(a)

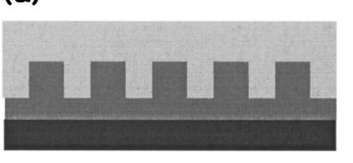

(b)
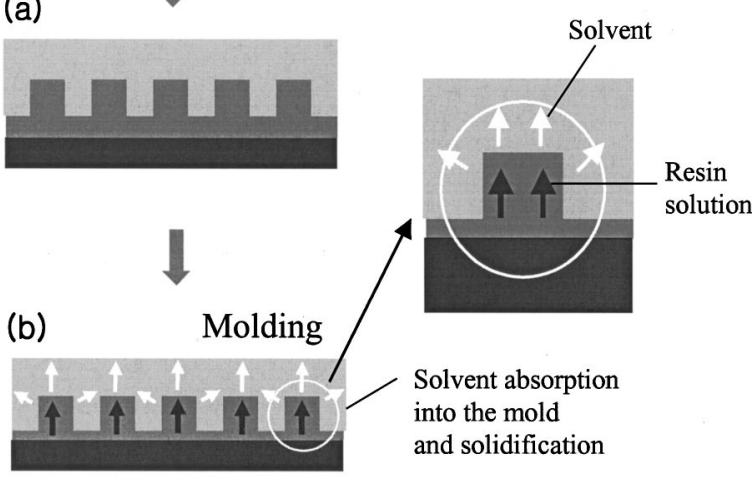

(c)

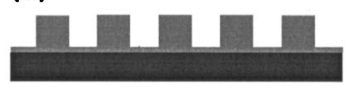

FIG. 1. Illustration of the soft molding method. In soft molding (a) an elastomeric mold is placed on a polymer film that is spin coated onto a substrate, which is then slightly pressed down to mold the pattern on the spin-coated polymer film. (b) After releasing the pressure, the whole structure is left undisturbed for a period of time for solidification, during which time the solvent in the molded structure is absorbed into the mold. (c) The mold is then removed.

min. During the soft molding, the solvent contained in the polymer film diffuses toward the interface due to the concentration gradient and is absorbed into the mold. The solvent then permeates through the mold and finally evaporates into the air. After the evaporation is completed, the mold is removed, thus finishing the soft molding.

The elastomeric mold in our experiment is PDMS (Sylgard 184, Dow Corning). PDMS molds were prepared by casting PDMS against masters having relief structures on the surface. A silicon wafer was used as the substrate for the coating of the polymer. The polymer used is a commercial novolac resin dissolved in propylene glycol mono ether acetate (PGMEA), which is a typical solvent for photoresists. The polymer was spin coated onto the wafer to $1.5-2.5 \mu \mathrm{m}$ thickness with $20-30 \mathrm{wt} \%$ polymer solution at $3000 \mathrm{rpm}$ for $15 \mathrm{~s}$. After soft molding the wet film, the molded threedimensional structures, which had been hardened by the time the mold is removed, were examined by scanning electron microscopy (SEM) and atomic-force microscopy (AFM).

Figure 2 shows SEM images of a three-dimensional structure that was formed by soft molding. Comparison of the master [Fig. 2(a)], from which the mold was prepared, with the polymer replica [Fig. 2(b)], obtained by soft molding, reveals that the original three-dimensional structure is well transferred to the polymer film without any distortion or defects in a single step. In the soft molding, the solvent in the molded polymer is continuously removed by absorption into PDMS mold at the interface between the mold and the molded polymer and replenished by solvent diffusion to the

Downloaded 13 Sep 2002 to 18.42.2.210. Redistribution subject
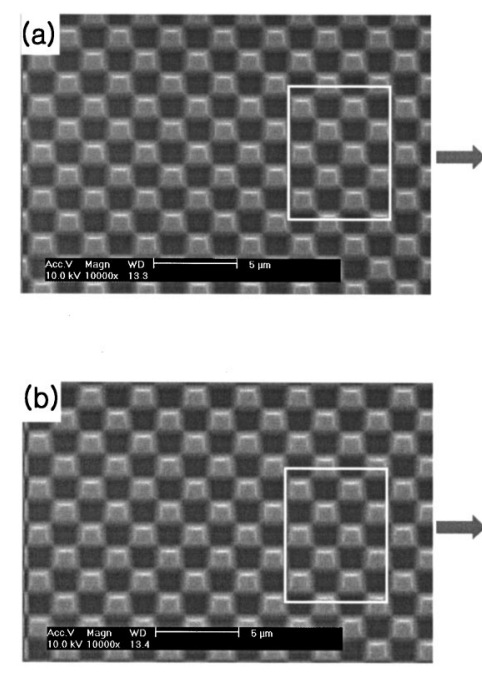

FIG. 2. SEM images of (a) the master used for soft molding and (b) the replica fabricated.

interface. The gradual and continuous replenishment of solvent and accompanying polymer to the interface assures the fidelity of the molded polymer structure. According to our experimental result, PDMS can absorb PGMEA up to 27 wt $\%$ of its weight. About $30 \mathrm{wt} \%$ of the absorbed solvent diffuses out into air in $1 \mathrm{~h}$. Although the dissipation rate is not high, the thickness of the polymer layer is negligible compared with that of PDMS mold such that the mold can more than accommodate all the solvent in the polymer film. For example, the solvent absorbed into the mold would amount to less than $0.1 \mathrm{wt} \%$ of the mold weight even if all the solvent in the wet polymer film were to be absorbed into the mold without any dissipation. The mold was used more than 20 times without any treatment.

The three-dimensional structure thus molded can be used as such since the molded polymer is hardened by the time the mold is removed. If baking is desired, it is carried out at the end of the molding with the mold still in place. To examine the effect of baking on the structural integrity, AFM images were taken for a sample structure obtained after soft molding and the same treated further by baking at $100^{\circ} \mathrm{C}$ for $1 \mathrm{~min}$. Although not shown here, the images revealed that the original molded structure remains unaltered even with baking.

To arrive at a condition under which soft molding can be effective, solvent absorption into the mold and evaporation from the polymer surface are modeled for their rates. Since the film of interest is quite thin, diffusion can be neglected. If it is assumed that the rate of solvent evaporation is proportional to the solvent concentration, then we have

$$
\frac{\partial M}{\partial t}=-k M,
$$

where $k$ is the proportionality constant and $M$ is the solvent concentration in terms of weight per unit area. Equation (1) gives

$$
M=M_{0} e^{-k t}
$$

The experimental data given in Fig. 3(a) show that the firstorder kinetics describes well the evaporation process. The solvent weight in Fig. 3(a) was determined experimentally 

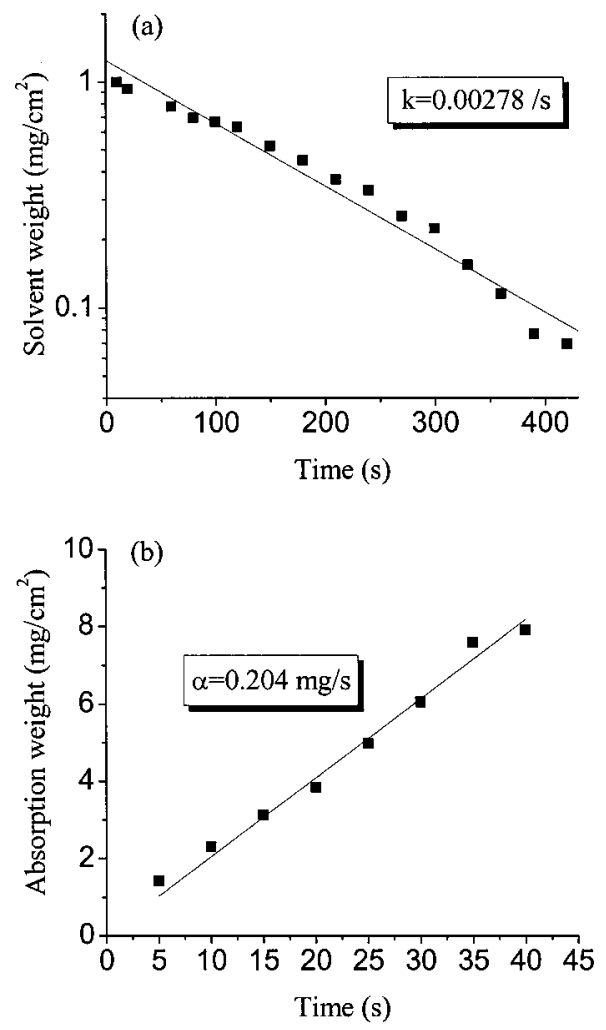

FIG. 3. Experimental determination of (a) evaporation parameter $k$ and (b) absorption parameter $\alpha$.

by dividing the weight of solvent evaporated by the exposed surface area. In the experiment for the rate of absorption by the mold, the mold was floated in a bath filled with the solvent. Since the solvent concentration is constant, the rate of absorption per exposed surface area is independent of the concentration such that

$$
\frac{\partial M_{a}}{\partial t}=-\alpha
$$

where $\alpha$ is a constant and $M_{a}$ is the concentration of the solvent per unit area in the mold. The data plotted in Fig. 3(b) show that the concentration $M_{a}$ increases linearly with time. For the soft molding to be successful, the rate of absorption should be larger than the rate of evaporation. Since the evaporation rate is exponential, whereas the absorption rate is linear, the initial rates should be used for the condition that the absorption rate be greater than the evaporation rate. It then follows from Eqs. (1) and (3) that

$$
\alpha>k M_{0} .
$$

In soft molding, the solvent is absorbed into the mold at the interface between the polymer film and the mold. Therefore, the value of $\alpha$ in the soft molding would be smaller than that of $\alpha$ determined in a bath of solvent. Therefore, the condition should be modified to

$$
\frac{\alpha}{k M_{0}} \gg 1 \text {. }
$$

For the experimental result in Fig. 3, novolac in PGMEA was used with PDMS as the mold, for which $\alpha$ is $0.204 \mathrm{mg} / \mathrm{s}$, $k$ is $0.00278 \mathrm{~s}^{-1}$, and $M_{0}=1.25 \mathrm{mg} / \mathrm{cm}^{2}$. It is seen that
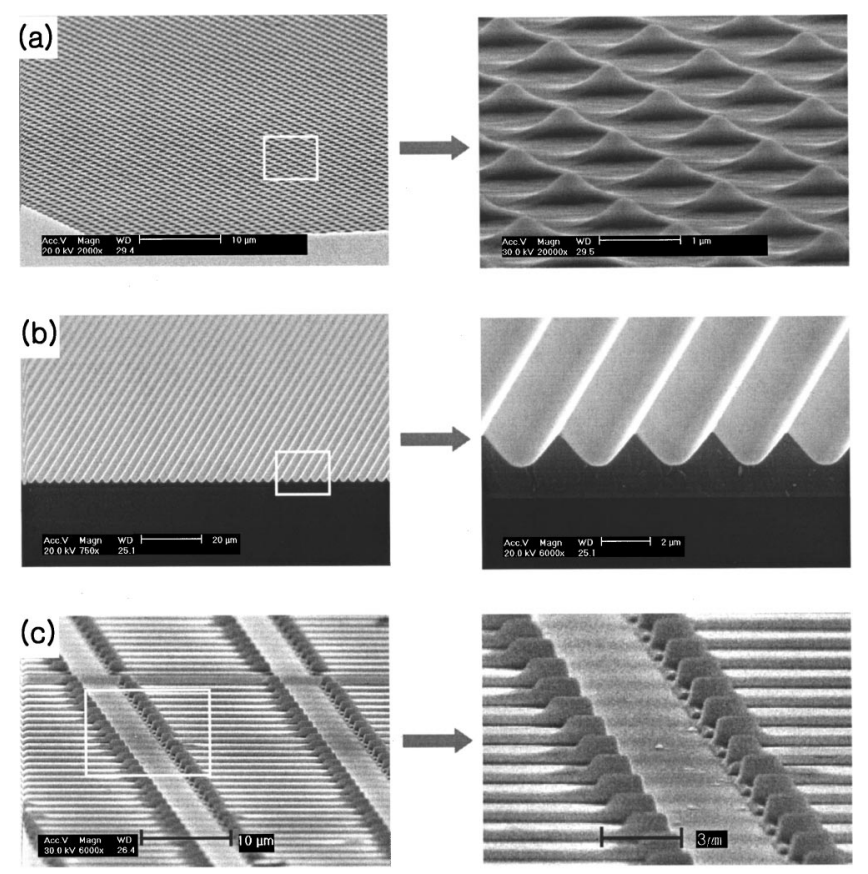

FIG. 4. Tilted SEM images of several three-dimensional structures fabricated by soft molding: (a) Circular cones, the enlarged part showing that the base diameter of the cone is approximately $1 \mu \mathrm{m}$; (b) rounded triangular channels with a period of approximately $3 \mu \mathrm{m}$; and (c) A three-level structure.

$\alpha /\left(k M_{0}\right)(=58.7)$ is much larger than unity. The same rate laws have been found to apply to other polymer systems such as polystyrene in toluene and polymethylmethacrylate in toluene. For instance, the values of $\alpha$ and $k$ for the system of polystyrene in toluene are $0.640 \mathrm{mg} / \mathrm{s}$ and $0.0312 \mathrm{~s}^{-1}$, respectively, such that $\alpha /\left(k M_{0}\right)(\sim 15) \gg 1$.

Figure 4 shows SEM images of several threedimensional structures formed by soft molding. Circular cones fabricated are shown in Fig. 4(a), the enlarged part revealing that the base diameter of the cone is approximately $1 \mu \mathrm{m}$. Rounded triangular channels with a period of approximately $3 \mu \mathrm{m}$ are given in Fig. 4(b). A three-level structure fabricated is shown in Fig. 4(c). These results demonstrate the effectiveness of soft molding in fabricating threedimensional structures.

Soft molding is an efficient and low-cost technique for fabricating three-dimensional structures. It is a simple method applicable to large-area patterning. In fact, we used the method to fabricate three-dimensional structures on areas of several square centimeters. Extension to larger areas should be quite possible.

${ }^{1}$ Y. Xia and G. M. Whitesides, Annu. Rev. Mater. Sci. 28, 153 (1998).

${ }^{2}$ E. Kim, Y. Xia, X.-H. Zhao, and G. M. Whitesides, Adv. Mater. 9, 651 (1997).

${ }^{3}$ K. E. Paul, T. L. Breen, J. Aizenberg, and G. M. Whitesides, Appl. Phys. Lett. 73, 2893 (1998).

${ }^{4}$ C. Marzolin, S. P. Smith, M. Prentiss, and G. M. Whitesides, Adv. Mater. 10, 571 (1998).

${ }^{5}$ S. Y. Chou, P. R. Krauss, and P. J. Renstrom, Appl. Phys. Lett. 67, 3114 (1995).

${ }^{6}$ H.-C. Scheer, H. Schult, T. Hoffmann, and C. M. Sotomayor Torres, J. Vac. Sci. Technol. B 16, 3917 (1998).

${ }^{7}$ P. Rai-Choudhury, Handbook of Microlithography, Micromachining, and Microfabrication (SPIE, Bellingham, WA, 1997), Vol. 2.

${ }^{8}$ D. Y. Khang and H. H. Lee, Appl. Phys. Lett. 76, 870 (2000). 